\title{
Lattice Boltzmann simulation of catalytic reactions
}

\author{
S. Arcidiacono* and J. Mantzaras ${ }^{\dagger}$ \\ Combustion Research, Paul Scherrer Institute, CH-5232 Villigen PSI, Switzerland
}

\begin{abstract}
I. V. Karlin
Aerothermochemistry and Combustion Systems Laboratory, ETH-Zurich, CH-8092 Zurich, Switzerland and School of Engineering Sciences, University of Southampton, SO17 1BJ Southampton, United Kingdom (Received 3 February 2008; revised manuscript received 10 September 2008; published 28 October 2008)
\end{abstract}

\begin{abstract}
A lattice Boltzmann model is developed to simulate finite-rate catalytic surface chemistry. Diffusive wall boundary conditions are established to account for catalytic reactions in multicomponent mixtures. Implementation of wall boundary conditions with chemical reactions is based on a general second-order accurate interpolation scheme. Results of lattice Boltzmann simulations for a four-component mixture with a global catalytic methane oxidation reaction in a straight channel are in excellent agreement with a finite volume Navier-Stokes solver in terms of both the flow field and species concentrations.
\end{abstract}

DOI: 10.1103/PhysRevE.78.046711

PACS number(s): 47.11.-j, 05.20.Dd

\section{INTRODUCTION}

Catalytic reactions are important in many industrial applications ranging from power generation and microreactors to pollutant abatement and chemical synthesis. In all these applications, reactions occur within a thin layer of porous medium wherein the catalyst is dispersed. The catalytic efficiency depends not only on the physical properties and loading of the catalyst, but also on the geometrical characteristics of the porous medium such as thickness, porosity, and tortuosity. The development of computational tools able to simulate flows at various scales with complex boundaries is necessary in order to understand the aforementioned heterogeneous reaction phenomena and subsequently to optimize the underlying processes.

The lattice Boltzmann (LB) method is an efficient simulation tool for flows in complex boundaries that, due to its kinetic origins, brings more physical insight in microflow simulations [1]. Therefore, LB is a good candidate for the simulation of flows in porous media with chemical reactions [2-4]. The application of LB to simulate catalytic surface reactions has also been reported in the literature [5-8]. However, most of the developed models applied the LB equations only to the bulk flow while the species transport was simulated separately, not accounting for the kinetics of each component. Though this kind of approach has been applied to different problems, it is strictly valid only if all but one species are tracers and it cannot capture some important mesoscopic phenomena as, for example, the dependence of the slip coefficient on the species concentration. Moreover, implementation of the diffusive wall boundary condition, which contrary to the bounce back (no-slip boundary condition) is more suited to the simulation of microchannels [1], is still missing in the modeling of surface reactions.

In Ref. [9] a multicomponent LB model was introduced for the simulation of realistic isothermal mixtures. Therein,

\footnotetext{
*arcisalvo@gmail.com

†ioannis.mantzaras@psi.ch

karlin.ilya@gmail.com
}

the distribution function of each species relaxes to equilibrium via a two-step path. The associated two relaxation times are related to an effective viscosity of each component and to the mixture-averaged diffusion coefficient (as summarized in Sec. II). The model satisfies the indifferentiability principle, i.e., when all the particles are mechanically equivalent the kinetic equations reduce to the single component case. In the macroscopic limit, the model recovers the Navier-Stokes and the Stefan-Maxwell diffusion equations (within the mixtureaverage diffusion approach), i.e., it can be employed as a standard macroscopic fluid solver. Moreover, the definition of an effective viscosity for each component as relaxation parameter and the use of proper diffusive wall boundary conditions enables the model to deal with microflows, with the correct prediction of the slip coefficient dependence on the species concentrations (as shown in [9]).

In the present paper, the aforementioned multicomponent nonreacting model is extended to simulate catalytic surface reactions. The diffusive boundary conditions [10] are generalized to account for chemical reactions. An efficient secondorder-accurate discretization scheme for the streaming step of populations with different molecular masses is introduced. In order to have a benchmark of the model with existing numerical tools, the model is applied to the simulation of catalytic reactions without accounting for intraphase (porous) diffusion. This approach is valid for thin catalyst coatings [11]. Results are compared with a finite volume NavierStokes solver already successfully applied on such problems [12].

The paper is organized as follows. Section II summarizes the theory of the multicomponent model. The catalytic model and its numerical implementation are reported in Sec. III. Finally, Sec. IV illustrates an application to catalytic channels and the good agreement of the predicted velocity and concentration profiles with the corresponding predictions of a finite volume reacting Navier-Stokes code.

\section{MULTICOMPONENT MODEL}

Some of the basic characteristics of the multicomponent LB model developed in Ref. [9] are reviewed in this section 


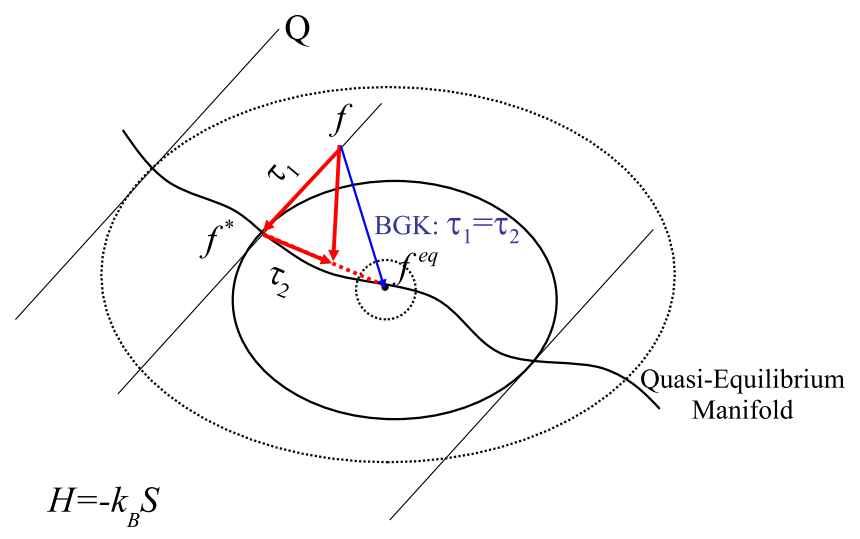

FIG. 1. (Color online) Schematic of the decomposition of motions near the quasiequilibrium manifold obtained by minimizing the $H$ function under some linear constraints $Q . \tau_{1}$, relaxation time from the initial state $f$ to the quasiequilibrium $f^{*}$. $\tau_{2}$, relaxation time from $f^{*}$ to the equilibrium $f^{\text {eq }}$. The standard BGK model is recovered when $\tau_{1}=\tau_{2}$. The closed curves define isolevels of $H=-k_{B} S$.

as they are necessary for the forthcoming development of the catalytic reaction model. Under the assumption of fast-slow decomposition of motions near the quasiequilibrium (QE) manifold $[13,14]$ (see also Fig. 1), the kinetic equation for species $j$ in a mixture of $M$ components is written as

$$
\begin{gathered}
\partial_{t} f_{j i}+c_{j i \alpha} \partial_{\alpha} f_{j i}=-\frac{1}{\tau_{1 j}}\left(f_{j i}-f_{j i}^{*}\right)-\frac{1}{\tau_{2 j}}\left(f_{j i}^{*}-f_{j i}^{\mathrm{eq}}\right)+F_{j i}, \\
j=1, \ldots, M, \quad i=0, \ldots, N,
\end{gathered}
$$

where $N$ is the number of the discrete lattice velocities $c_{j i \alpha}, \alpha=\{x, y\}$ is the spatial direction, $\tau_{1 j}$ and $\tau_{2 j}$ are the relaxation times of each species $j$ from the initial state $f$ to the QE $f^{*}$ and from $f^{*}$ to equilibrium $f^{\mathrm{eq}}$, respectively; $F_{j i}$ is a forcing term acting on species $j$. The forcing term is required in order to maintain the momentum balance when the mixture-average approximation for diffusion coefficients is employed (see Ref. [9] for details). In Eq. (1) and henceforth, Einstein's summation convention is applied only to the spatial direction $\alpha$.

For the two-dimensional nine-velocity LB model (D2Q9) considered herein, the discrete velocity set for each component $j$ is

$$
c_{j i}= \begin{cases}c_{j}\left\{\cos \left(\frac{\pi(i-1)}{2}\right), \sin \left(\frac{\pi(i-1)}{2}\right)\right\}, & i=0, \\ c_{j} \sqrt{2}\left\{\cos \left(\frac{\pi(2 i-9)}{4}\right), \sin \left(\frac{\pi(2 i-9)}{4}\right)\right\}, & i=5-8,\end{cases}
$$

where $c_{j}=\sqrt{1 / m_{j}}$ is the magnitude of the lattice vector in LB units (see Sec. III for a detailed explanation on the unit scaling), and $m_{j}$ is the molecular mass of component $j$.

The fast-slow decomposition implies, by construction, the inequality $\tau_{1 j} \leqslant \tau_{2 j}$ that must be respected in order to ensure the $H$ theorem $[9,13,15-17]$, i.e., the entropy $S$ of the system is always growing thus ensuring thermodynamic consistency.
The equilibrium distribution function $f^{\mathrm{eq}}$ is obtained by maximizing the entropy function under the constraints of conserved density $\rho_{j}$ of component $j$, and total momentum $\mathbf{J}$ of the mixture $[9,15,16,18,19]$

$$
\begin{aligned}
f_{j i}^{\mathrm{eq}}\left(\rho_{j}, \mathbf{U}\right)= & \rho_{j} W_{i} \prod_{\alpha=x, y}\left(\frac{2 c_{j}-\sqrt{c_{j}^{2}+3 U_{\alpha}^{2}}}{c_{j}}\right) \\
& \times\left(\frac{2 U_{\alpha}+\sqrt{c_{j}^{2}+3 U_{\alpha}^{2}}}{c_{j}-U_{\alpha}}\right)^{c_{j i \alpha} / c_{j}}
\end{aligned}
$$

where $U_{\alpha}=J_{\alpha} / \rho$ is the velocity of the mixture in the $\alpha$ direction, $\rho=\sum_{j}^{M} \rho_{j}$ is the mixture density, and

$$
W_{i}= \begin{cases}\frac{4}{9}, & i=0, \\ \frac{1}{9}, & i=1-4, \\ \frac{1}{36}, & i=5-8 .\end{cases}
$$

Note that the ratio $c_{j i \alpha} / c_{j}$ assumes always the values $[-1,0,1]$ as in the single component case.

The quasiequilibrium distribution functions $f^{*}$ are obtained by assuming that in the multicomponent mixture some particular moments approach equilibrium slower than the other moments. The relevant moments that characterize the momentum transport of one component in a mixture due to viscosity and diffusion are the pressure tensor $P_{j \alpha \beta}$ and the momentum difference $\mathbf{J}-\mathbf{J}_{j}$, respectively. Maximizing the entropy [as previously done to recover Eq. (3)] with the further condition that either $P_{j \alpha \beta}$ or $\mathbf{J}-\mathbf{J}_{j}$ are conserved, two different QE distribution functions are established. Using alternatively one of the two $f^{*}$, it is possible to recover two different complementary models, which will be further denoted as model I and model II.

As shown in Ref. [9], the expression of the QE of model I (obtained by considering $P_{\alpha \beta}$ as slow variable) is somewhat complicated, and, from an implementation point of view, a good approximation of such QE is the Grad's distribution function [20],

$$
\begin{aligned}
f_{j i}^{*}\left(\rho_{j}, J_{\alpha}, P_{j \alpha \beta}\right)= & W_{i}\left(\rho_{j}+\frac{J_{\alpha} c_{j i \alpha}}{c_{j s}^{2}}\right. \\
& \left.+\frac{1}{2 c_{j s}^{4}}\left(P_{j \alpha \beta}-\rho_{j} c_{j s}^{2} \delta_{\alpha \beta}\right)\left(c_{j i \alpha} c_{j i \beta}-c_{j s}^{2} \delta_{\alpha \beta}\right)\right),
\end{aligned}
$$

where $c_{j s}=c_{j} / \sqrt{3}$.

Finally the QE for model II is the same as the equilibrium distribution equation (3) where the velocity of each component $\mathbf{U}_{j}=\mathbf{J}_{j} / \rho_{j}$ is used instead of the total mixture velocity,

$$
f_{j i}^{*}\left(\rho_{j}, \mathbf{U}_{j}\right)=f_{j i}^{\mathrm{eq}}\left(\rho_{j}, \mathbf{U}_{j}\right) .
$$

For both models, the Chapman-Enskog expansion allows for identifying the $2 M$ relaxation times,

$$
\tau_{1 j}=\tau_{\mathrm{diff}, j} \quad \text { and } \tau_{2 j}=\tau_{\mathrm{visc}, j} \quad \text { model I, }
$$




$$
\tau_{1 j}=\tau_{\mathrm{visc}, j} \quad \text { and } \tau_{2 j}=\tau_{\mathrm{diff}, j} \quad \text { model II, }
$$

where $\tau_{\mathrm{visc}, j}$ and $\tau_{\mathrm{diff}, j}$ are two time constants that depend on an effective viscosity of component $j$ in the mixture, $\mu_{\mathrm{eff}, j}$, and on the mixture-average diffusion coefficient, $D_{j m}$, respectively,

$$
\tau_{\mathrm{visc}, j}=\frac{\mu_{\mathrm{eff}, j}}{P}, \quad \tau_{\mathrm{diff}, j}=\frac{\rho_{j}}{P X_{j}} D_{j m},
$$

where $P$ is the pressure and $X_{j}$ is the mole fraction of component $j$.

The effective viscosity $\mu_{\mathrm{eff}, j}$ is a function of the actual viscosity $\mu_{j}$ of component $j$ and of the concentration of all components in the mixture. As already discussed in [9], we use the formula proposed by Wilke [21] and then modified by Bird [22],

$$
\begin{gathered}
\mu=\sum_{j}^{M} X_{j} \mu_{\mathrm{eff}, j}, \quad \mu_{\mathrm{eff}, j}=\frac{\mu_{j}}{\sum_{k}^{M} X_{k} \Phi_{j k}}, \\
\Phi_{j k}=\frac{1}{\sqrt{8}}\left(1+\frac{m_{j}}{m_{k}}\right)^{-1 / 2}\left[1+\left(\frac{\mu_{j}}{\mu_{k}}\right)^{1 / 2}\left(\frac{m_{k}}{m_{j}}\right)^{1 / 4}\right]^{2},
\end{gathered}
$$

where $\mu$ is the viscosity of the mixture. The mixture-average diffusion coefficient $D_{j m}$ is defined as

$$
D_{j m}=\frac{1-Y_{j}}{\sum_{k \neq j}^{M} X_{k} / \mathcal{D}_{j k}},
$$

with $Y_{j}$ is the mass fraction of component $j$ and $\mathcal{D}_{j k}$ is the binary diffusion coefficient [23].

The physical meaning of the proposed model becomes clear when the Schmidt number of component $j$ is defined as

$$
\mathrm{Sc}_{j}=\frac{\mu_{\mathrm{eff}, j}}{\rho D_{j m}}
$$

It is possible to express $\mathrm{Sc}_{j}$ in terms of relaxation times

$$
\begin{aligned}
\mathrm{Sc}_{j} & =\frac{\mu_{\mathrm{eff}, j}}{\rho D_{j m}} \\
& =\frac{\tau_{\mathrm{visc}, j}}{\tau_{\mathrm{diff}, j}} \frac{Y_{j}}{X_{j}} \\
& =\left\{\begin{array}{l}
\frac{\tau_{2 j}}{\tau_{1 j}} \frac{Y_{j}}{X_{j}} \geqslant \frac{Y_{j}}{X_{j}}=\mathrm{Sc}_{j}^{*} \quad \text { if } \tau_{\mathrm{visc}, j}>\tau_{\mathrm{diff}, j} \quad \text { (model I), } \\
\frac{\tau_{1 j}}{\tau_{2 j}} \frac{Y_{j}}{X_{j}} \leqslant \frac{Y_{j}}{X_{j}}=\mathrm{Sc}_{j}^{*} \quad \text { if } \tau_{\mathrm{visc}, j}<\tau_{\mathrm{diff}, j} \quad \text { (model II), }
\end{array}\right.
\end{aligned}
$$

where $\mathrm{Sc}_{j}^{*}$ is a reference Schmidt number. This implies that in model I (where the component $P_{j \alpha \beta}$ is considered as the slow variable) $\mathrm{Sc}_{j} \geqslant \mathrm{Sc}_{j}^{*}$ and the momentum transport is dominated by viscosity. On the other hand, in model II the slow variable is $\mathbf{J}-\mathbf{J}_{j}$ and $\mathrm{Sc}_{j} \leqslant \mathrm{Sc}_{j}^{*}$. With this kind of construction it is possible to simulate mixtures at arbitrary Schmidt numbers and, at the same time, the thermodynamic consistency is preserved.

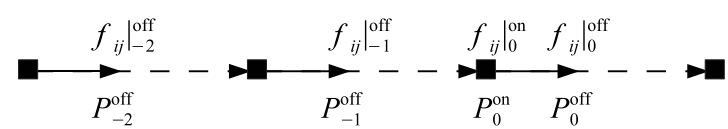

FIG. 2. Streaming step. The populations corresponding to the lightest component are streamed on-lattice (dashed line). Populations corresponding to larger molecular masses are streamed offlattice (continuous line), the corresponding on-lattice values are calculated by interpolating the off-lattice neighbors.

It is clarified that for both models the indifferentiability principle is satisfied when all the molecular weights $m_{j}$ are the same, and $\tau_{1 j}=\tau_{2 j}=\tau$, where $\tau$ is a constant for $j$ $=1, \ldots, M$ in Eq. (1), see also Fig. 1 .

\section{CATALYTIC SURFACE REACTION MODEL AND IMPLEMENTATION}

In this section details of the implementation of a generic multicomponent LB model with surface reactions are given. The streaming of populations having different molecular masses is initially discussed and a second-order accurate scheme is suggested, followed by the implementation of reactive wall boundary conditions. Finally, the scaling between $\mathrm{LB}$ and physical units is clarified.

\section{A. Interpolation scheme}

Compared to standard single-component LB schemes, the streaming step in a multicomponent LB model is more complicated due to the different masses of components that in turn imply different lattice speeds $[9,15,16,24]$. As shown in Fig. 2 (dashed arrows) the populations corresponding to the lightest component are streamed on-lattice as in the onecomponent case $\left(c_{1}=1\right)$. On the other hand, heavier components with a slower lattice speed cannot stream from one lattice node to another (off-lattice streaming) and an interpolation step is needed. The choice of a proper interpolation scheme is important since the scheme can introduce numerical diffusion, irrespective of the numerical order [25]. Moreover, in order to correctly predict the surface reaction rates, which depend on the density gradients at the catalytic wall, it is important that the numerical scheme retains its accuracy near the solid boundaries. Previous implementations $[9,15,16]$ adopted various interpolation schemes, such as the upwind Lagrangian interpolation [24] or the volumetric interpolation [26]. Both schemes have some advantages and drawbacks: The former is computationally efficient, but it does not allow for an easy wall boundary implementation, while the latter is based on some physical considerations (mass and momentum conservation in a volume), but it is computational expensive. For these reasons a different scheme is herein developed.

After the (off-lattice) streaming step, the populations on the lattice are unknown (Fig. 2). By expressing the unknown population $\left.f_{i j}\right|_{0} ^{\text {on }}$ at a lattice node $P_{0}^{\text {on }}$ as a function of the population $\left.f_{i j}\right|_{-1} ^{\text {off }}$ at the off-lattice upwind position $P_{-1}^{\text {off }}$ through a Taylor series expansion, the following is obtained: 

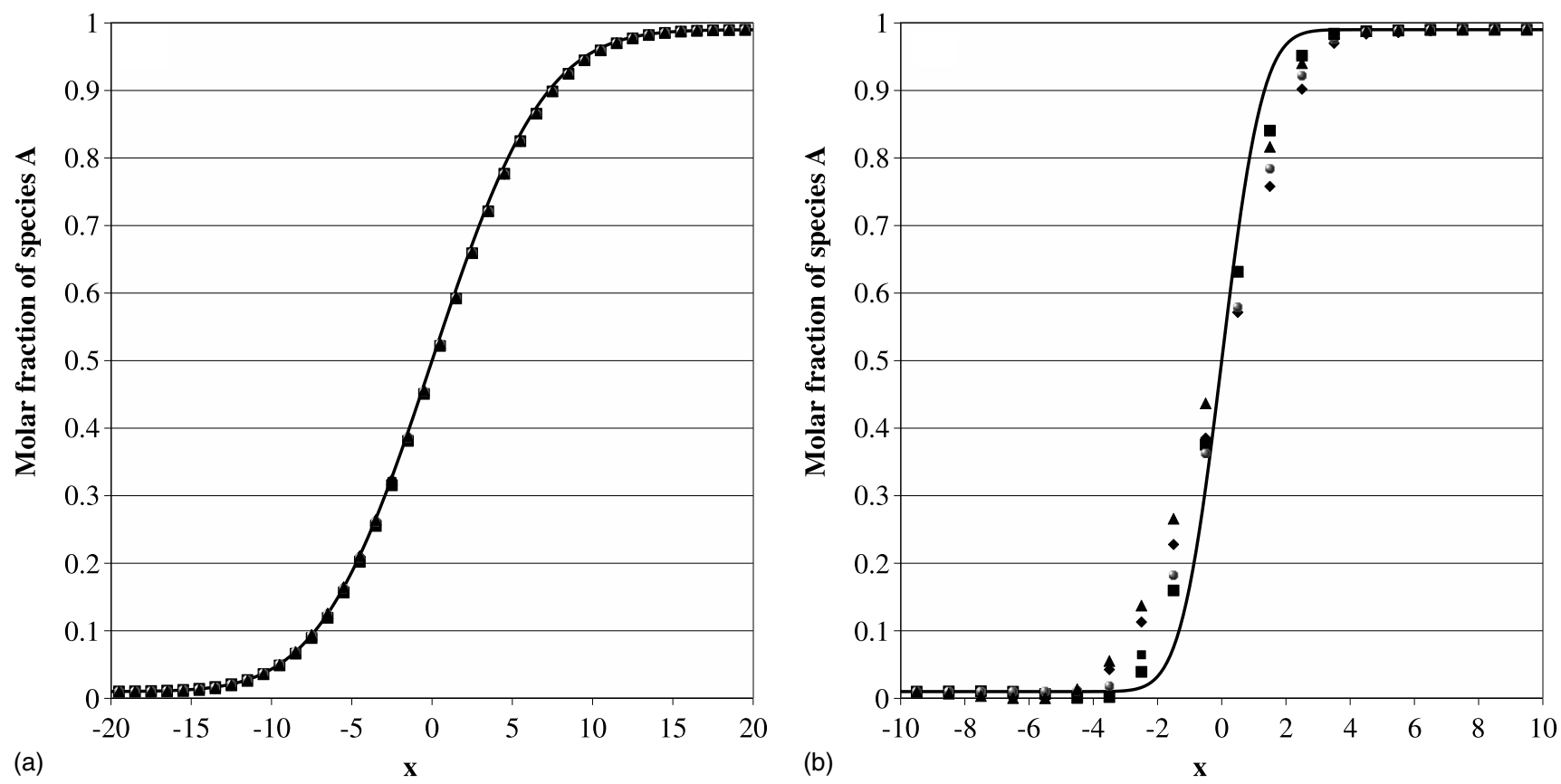

FIG. 3. Comparison among four different discretization schemes (symbols) after 500 time steps and the corresponding analytical solution (solid lines) for the diffusion of two components $(A, B)$ of different molecular weights (mass ratio 20). Initial composition: $1 \% A-99 \% B$ at $x<0,99 \% A-1 \% B$ at $x \geqslant 0$. Triangles, present work; circles, flux limiters [27,28]; squares, volumetric scheme [26]; diamonds, second-order upwind [24]. Panel (a) diffusion coefficient $D=3 \times 10^{-2}$ (in scaled LB units, see Sec. III C), panel (b) $D=10^{-3}$.

$$
\left.f_{i j}\right|_{0} ^{\text {on }}=\left.f_{i j}\right|_{-1} ^{\text {off }}+f_{i j}^{\prime}||_{-1}^{\text {off }}\left(1-p_{j}\right)+\left.f_{i j}^{\prime \prime}\right|_{-1} ^{\text {off }} \frac{\left(1-p_{j}\right)^{2}}{2}+O\left(1-p_{j}\right)^{3}
$$

where

$$
p_{j}=\left\|c_{j}\right\| d t
$$

is the distance that a population travels at one time step with speed $c_{j} ;\left.\quad f_{i j}^{\prime}\right|_{-1} ^{\text {off }}$ and $\left.f_{i j}^{\prime \prime}\right|_{-1} ^{\text {off }}$ are the first and second spatial derivatives at the off-lattice position $P_{-1}^{\text {off }}$, respectively, which can be evaluated with the second-order finite difference formulas

$$
\begin{gathered}
\left.f_{i j}^{\prime}\right|_{-1} ^{\text {off }}=\frac{\left.f_{i j}\right|_{0} ^{\text {off }}-\left.f_{i j}\right|_{-2} ^{\text {off }}}{2}, \\
\left.f_{i j}^{\prime \prime}\right|_{-1} ^{\text {off }}=\left.f_{i j}\right|_{0} ^{\text {off }}+\left.f_{i j}\right|_{-2} ^{\text {off }}-\left.2 f_{i j}\right|_{-1} ^{\text {off }},
\end{gathered}
$$

far from the boundaries or

$$
\begin{aligned}
& \left.f_{i j}^{\prime}\right|_{-1} ^{\text {off }}=\frac{\left.4 f_{i j}\right|_{0} ^{\text {off }}-\left.f_{i j}\right|_{+1} ^{\text {off }}-\left.3 f_{i j}\right|_{-1} ^{\text {off }}}{2}, \\
& \left.f_{i j}^{\prime \prime}\right|_{-1} ^{\text {off }}=-\left.2 f_{i j}\right|_{0} ^{\text {off }}+\left.f_{i j}\right|_{+1} ^{\text {off }}+\left.f_{i j}\right|_{-1} ^{\text {off }},
\end{aligned}
$$

near the boundaries where the population $f_{-2}^{\text {off }}$ does not exist. By substituting Eqs. (15) [respectively, Eqs. (16)] in Eq. (13) a generic off-lattice streaming step is obtained. This interpolation scheme is equivalent to a second-order Lax-Wendroff discretization scheme of the convective term in Eq. (1), but compared to schemes proposed in the past that used a Taylor expansion around $P_{0}^{\text {off }}$ (see the example reported in [25]) the present scheme is obtained by expanding around $P_{-1}^{\text {off }}$, and thus includes more upwind information. It is clear that when $p=1$, the standard on-lattice LB streaming procedure is recovered.

The numerical accuracy of the present interpolation scheme was tested by comparing it with the two aforementioned schemes (the second-order Lagrangian upwind [24] and the volumetric interpolation scheme [26]), with the flux limiter scheme proposed by Sofonea et al. [27,28] and also with the corresponding analytical solution for the binary diffusion of species with different mass ratios as described in [16] (see Fig. 3). The four interpolation schemes lead to the correct result for well-resolved grids. As reported in [27], all schemes become less accurate when the diffusion coefficient is small and/or when the grid is coarse. Moreover, spurious density oscillations can be induced by second-order interpolation schemes (upwind, volumetric, and the present scheme). All of the above-mentioned schemes can be implemented and applied to the proposed multicomponent kinetic equations. However, the interpolation herein proposed has several advantages: It is second-order accurate not only in the interior, but also close to the boundaries allowing for a correct evaluation of the mass fluxes at the wall (necessary to further evaluate the catalytic reaction rates); it is more local than other schemes; it is easy to implement at the boundaries; and it involves only three lattice nodes while other schemes use up to nine. Consequently, the streaming step of the present implementation is computationally practical: In the diffusion test the scheme performed about 2 times as fast as the volumetric scheme and about 1.5 times faster than the flux limiters scheme. 


\section{B. Wall boundary conditions for surface reactions}

Diffusive boundary conditions [10] are herein adapted in order to include surface chemistry. Imposing mass conservation for each component at the wall, the unknown incoming mass fluxes to the computational domain, $\Phi_{j}^{\text {in }}$, must balance the outgoing fluxes, $\Phi_{j}^{\text {out }}$, plus the reaction rate terms $\dot{S}_{j}$ that depends on the local species concentration $Y_{j}$,

$$
\Phi_{j}^{\text {in }}-\Phi_{j}^{\text {out }}=\dot{S}_{j} .
$$

Rewriting the fluxes in Eqs. (17) in terms of populations, we have

$$
\sum_{i, f_{j i} c_{j i \alpha^{n} n_{\alpha}>0}}\left|f_{j i} c_{j i \alpha} n_{\alpha}\right|-\sum_{i, f_{j i} c_{j i \alpha^{\prime} n_{\alpha}<0}}\left|f_{j i} c_{j i \alpha} n_{\alpha}\right|=\dot{S}_{j},
$$

where $n_{\alpha}$ is the component in the $\alpha$ direction of the inward unit vector normal to the wall, and $f_{j i} c_{j i \alpha}$ is the mass flux of component $j$ transported by the velocity $i$ in the $\alpha$ direction. In order to evaluate the incoming populations, two more equations are required for each component. Considering that the populations have sufficient time to equilibrate with the wall temperature, the resulting velocity distributions are considered to be Maxwellian,

$$
\frac{f_{j i}}{f_{j i}^{\mathrm{eq}}\left(\rho=1, U_{w}\right)}=\rho_{w},
$$

where $f_{j i}^{\mathrm{eq}}\left(\rho=1, U_{w}\right)$ are the equilibrium distribution functions evaluated at a constant unit density and wall velocity $U_{w}$, and $\rho_{w}$ is the density at the wall. The evaluation of $f_{j i}^{\mathrm{eq}}\left(\rho=1, U_{w}\right)$ at a reference unit density is necessary, contrary to the single component case where the unknown wall density is not required to evaluate the incoming populations, since the production term $\dot{S}_{j}$ is a function of the concentrations at the wall. Solving the systems of Eqs. (17) and (19), we have

$$
f_{j i}=f_{j i}^{\mathrm{eq}}\left(\rho=1, U_{w}\right) \frac{\sum_{i, f_{j i} c_{j i \alpha^{n}}{ }<0}\left|f_{j i} c_{j i \alpha} n_{\alpha}\right|+\dot{S}_{j}}{\sum_{i, f_{j i}^{\mathrm{eq}} c_{j i \alpha^{\prime}} n>0}\left|f_{j i}^{\mathrm{eq}}\left(\rho=1, U_{w}\right) c_{j i \alpha} n_{\alpha}\right|} .
$$

Equation (20) provides a generic expression of diffusive reacting wall boundary conditions. Since $\dot{S}_{j}$ depends on the local species concentration that is initially unknown, an iterative procedure is required at each time step to evaluate it, as described below.

The practical implementation of the wall boundary condition requires particular attention. As already reported in [29], the actual wall position is located at $y=0.5$ : The incoming flux $\Phi_{j}^{\text {in }}$ should be considered as generated in external "ghost" nodes (see Fig. 4); this flux reaches the wall at $t$ $=d t / 2$ (i.e., wall position at $y=0.5$ ) where it thermalizes with the outgoing flux $\Phi_{j}^{\text {out }}$. Finally, at time $t=d t, \Phi_{j}^{\text {in }}$ reaches the nodes at $y=1$. Since in the actual multicomponent case the lattice velocities are not the same for each component, some additional effort is required in order to ensure second-order accuracy at the wall boundary. As stated before, the outgoing

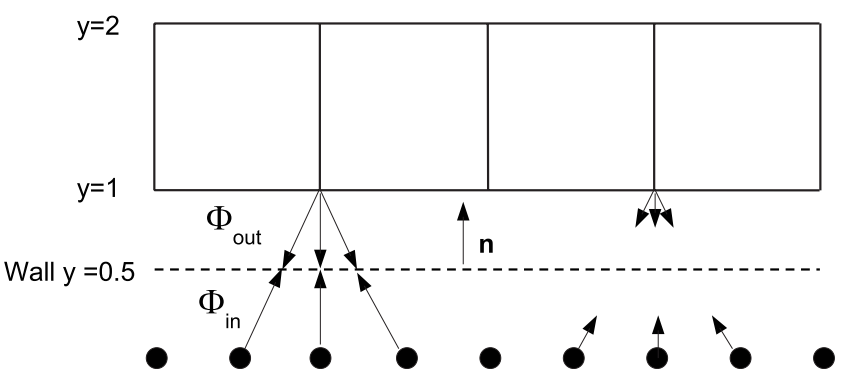

FIG. 4. Schematic of wall boundary condition: The incoming flux $\Phi_{j}^{\text {in }}$ is equilibrating with the outgoing $\Phi_{j}^{\text {out }}$ at $y=0.5$.

flux $\Phi_{j}^{\text {out }}$ is needed at the wall node $y=0.5$ at time $d t=0.5$. The outgoing populations $\left.f_{i j}\right|_{0.5} ^{\text {out }}$ are extrapolated to the wall by using Eqs. (13),

$$
\begin{aligned}
\left.f_{i j}\right|_{0.5} ^{\text {out }}= & \left.f_{i j}\right|_{1} ^{\text {off }}+\left.f_{i j}^{\prime}\right|_{1} ^{\text {off }}\left(0.5-\frac{p_{j}}{2}\right)+\left.f_{i j}^{\prime \prime}\right|_{1} ^{\text {off }} \frac{1}{2}\left(0.5-\frac{p_{j}}{2}\right)^{2} \\
& +O\left(0.5-\frac{p_{j}}{2}\right)^{3}
\end{aligned}
$$

where $\left.f_{i j}^{\prime}\right|_{1} ^{\text {off }}$ and $\left.f_{i j}^{\prime \prime}\right|_{1} ^{\text {off }}$ are evaluated from Eqs. (16). Equations (20) can be subsequently used to evaluate the unknown thermalized populations at the wall $\left.f_{i j}\right|_{0.5} ^{\text {in }}$. By using Eqs. (13), $\left.f_{i j}\right|_{0.5} ^{\text {in }}$ can be extrapolated to the "ghost" nodes,

$$
\begin{aligned}
\left.f_{i j}\right|_{0.5} ^{\text {in }}= & \left.f_{i j}\right|_{0} ^{\text {off }}+\left.f_{i j}^{\prime}\right|_{0} ^{\text {off }}\left(0.5-\frac{p_{j}}{2}\right)+\left.f_{i j}^{\prime \prime}\right|_{0} ^{\text {off }} \frac{1}{2}\left(0.5-\frac{p_{j}}{2}\right)^{2} \\
& +O\left(0.5-\frac{p_{j}}{2}\right)^{3}
\end{aligned}
$$

that must be solved for $\left.f_{i j}\right|_{0} ^{\text {off }}$. Finally, Eqs. (13) and (16) can be used again to stream $\left.f_{i j}\right|_{0} ^{\text {off }}$ to the nodes at $y=1$.

Another implementation difficulty appears in Eqs. (18), which depend on the reaction terms $\dot{S}_{j}$ that are in turn a function of the local concentrations at the wall (unknown $a$ priori). The problem is solved by assuming initially that the concentration at the wall is the same as that at node $y=1 ; \dot{S}_{j}$ are then evaluated, and Eqs. (18) are applied to evaluate the known populations at the wall. Finally, Eqs. (19) are used to compute the resulting density (concentration) at wall. The procedure is then iterated until the relative difference between the assumed density at the wall and the one calculated with Eqs. (19) is less than $10^{-6}$. Note that the iterative algorithm to solve the implicit dependence on the density at the wall is not a numerical artifact but it rather reflects the physics of the reaction in a infinitely thin layer of catalyst, wherein there is a direct coupling of reaction rates and species populations [as manifested by Eq. (17)].

\section{Dimensions}

Chemical reactions and transport properties are managed with the CHEMKIN package $[23,30]$. The integration of CHEMKIN libraries into the LB code requires a proper scaling of the problem variables in LB units for the correct evaluation of mixture properties and reaction rates. Once the char- 


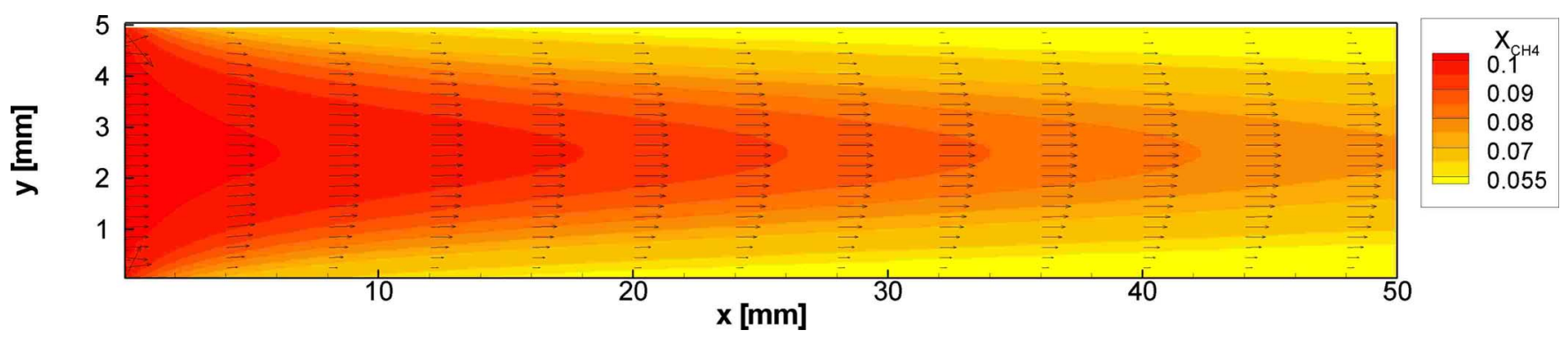

FIG. 5. (Color online) Computational domain. Velocity vectors and $\mathrm{CH}_{4}$ isomole-fraction profiles.

acteristic length of the domain $L$ and the lattice resolution $L_{\mathrm{LB}}$ have been chosen, the length scale is set to

$$
L^{*}=\frac{L}{L_{\mathrm{LB}}},
$$

which is the physical length in lattice units. The velocity scale $v^{*}$ is defined such that once the velocities are scaled into LB units, the lattice velocity of the lightest component of mass $m_{1}$ is $c_{1}=1$ (implying that for all the other lattice velocities $c_{j} \leqslant 1$ ),

$$
v^{*}=\sqrt{\frac{3 R T_{0}}{m_{1}}}
$$

It is noted that the velocity scale $v^{*}$ is not equal to the speed of sound, but depends on the employed numerical scheme: For the current D2Q9 implementation, a factor of $\sqrt{3}$ is required in Eq. (24) in order to have a correct reference velocity and to recover the correct $\mathrm{Kn}$ number $[1,9]$. The time scale is immediately deduced as

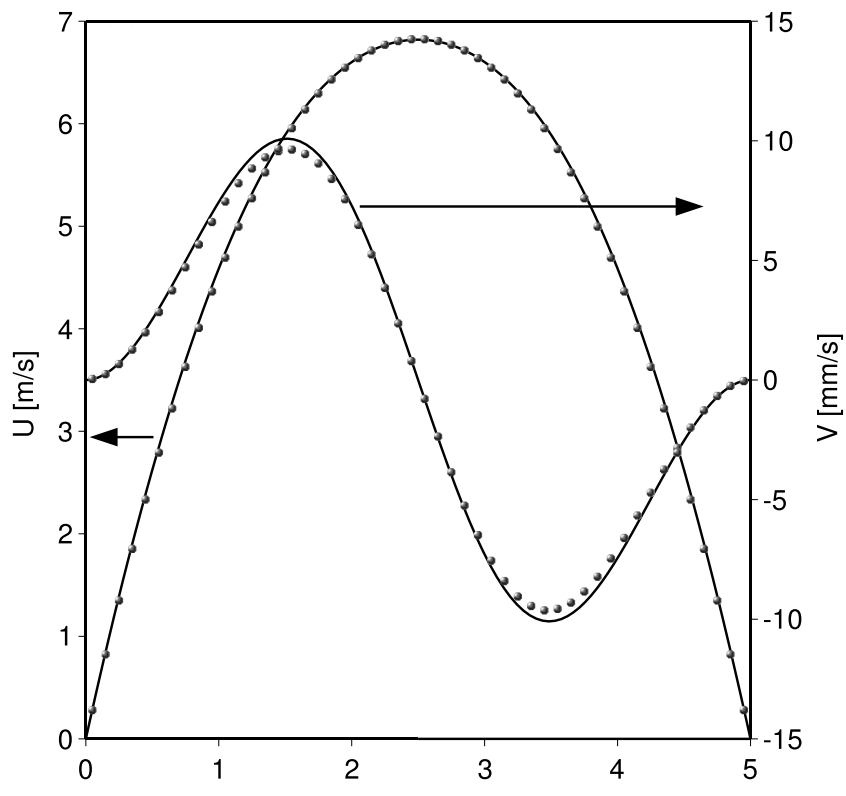

(a)

$$
\mathrm{y}[\mathrm{mm}]
$$

$$
t^{*}=\frac{L^{*}}{v^{*}} .
$$

Finally, the density scale $\rho^{*}$ is chosen such that the LB density is numerically equal to physical SI units, i.e., $\rho *$ $=1\left[\mathrm{~kg} \mathrm{~m}^{-3}\right]$. All these reference values are used to reduce CHEMKIN library outputs into LB units and vice versa.

\section{APPLICATION}

The model is tested on the simulation of a planar catalytic channel having $5 \mathrm{~mm}$ height and $50 \mathrm{~mm}$ length (Fig. 5). For the sake of simplicity, a one-step reaction valid for fuel-lean combustion is herein considered to simulate the conversion of fuel-lean methane on a catalytic platinum surface [12]:

$$
\mathrm{CH}_{4}+2 \mathrm{O}_{2} \rightarrow 2 \mathrm{H}_{2} \mathrm{O}+\mathrm{CO}_{2}, \quad k=A \exp \left(-E_{a} / R T_{w}\right),
$$

where $k$ is the reaction rate constant, $A=1.27 \times 10^{5} \mathrm{~cm} / \mathrm{s}$, and $E_{a}=84 \mathrm{~kJ} / \mathrm{mol}$ is the apparent activation energy. The reaction rate $\dot{S}$ of this global step is, under lean conditions,

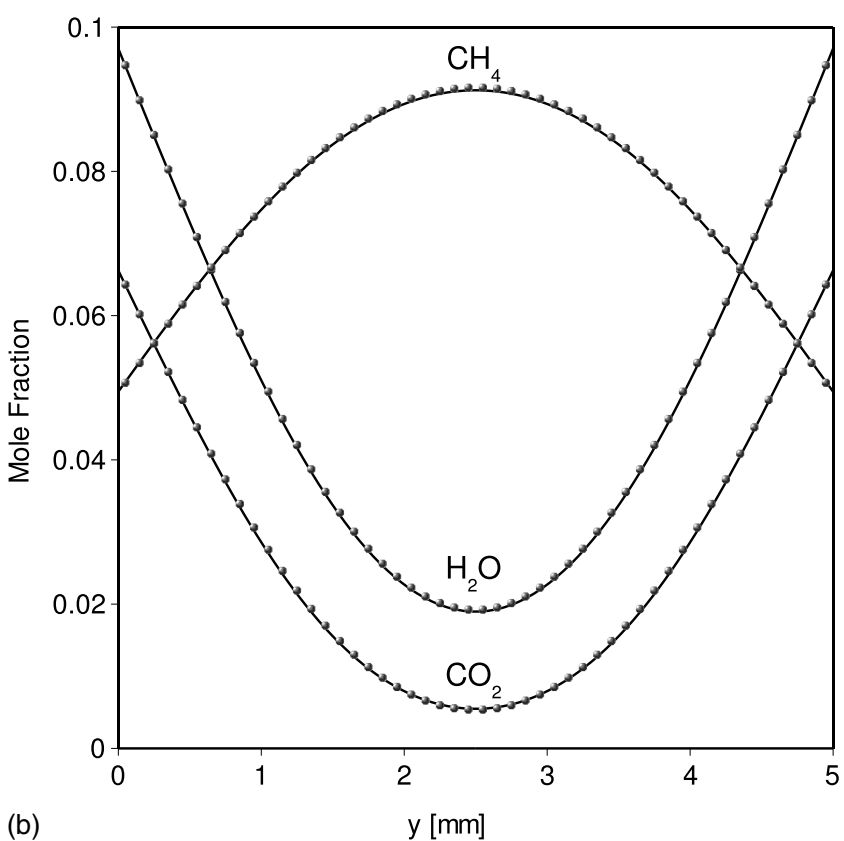

(b) $y[\mathrm{~mm}]$

FIG. 6. Comparison, in a cross section at the middle of the channel, between the LB code $(500 \times 50$ grid nodes $)$ and a two-dimensional steady finite volume code. Panel (a), velocity profiles. Panel (b), concentration profiles. Continuous lines CFD, symbols LB predictions. 
only a function of methane concentration with a first-order dependence, $\dot{S}=k\left[\mathrm{CH}_{4}\right]$. The calculations are performed at constant temperature of $1200 \mathrm{~K}$ and atmospheric pressure. Equation (20) is also used to evaluate the unknown incoming particle flux at the inlet, by setting the source term $\dot{S}_{j}$ equal to the incoming mass flux of each component $j$. This type of inlet boundary condition ensures that the incoming mass flux of each component is constant, i.e., the concentration at the inlet is fixed. The resulting imposed inlet mole fractions, velocity and pressure are $X_{\mathrm{O}_{2}}=90 \%, X_{\mathrm{CH}_{4}}=10 \%, U$ $=4.6 \mathrm{~m} / \mathrm{s}$, and $P=1.08 \mathrm{bar}$, respectively. At the outlet, all the populations (known and unknown) are replaced with the equilibrium distributions calculated in accordance to the velocity and density at the previous node. At time $t=0$, the molar concentrations inside the channel are set equal to the ones at the inlet, and the concentration of the product species $\left(\mathrm{H}_{2} \mathrm{O}\right.$ and $\left.\mathrm{CO}_{2}\right)$ is initialized to $10^{-14}$. The grid resolution is set to $500 \times 50$ nodes. Results are compared with a steady finite volume code [31] that has a well-resolved flow (400 $\times 120$ grid points).

In Figs. 6(a) and 6(b) the velocity and concentration profiles are provided for both codes at a cross section in the middle of the channel $(x=25 \mathrm{~mm})$. Both the axial and the transverse velocity components are well captured, despite the fact that the latter is two orders of magnitude smaller than the former. The concentration profiles are also well predicted, clearly showing that both the diffusion equations and the reaction rates are correctly reproduced. It is emphasized that the wall concentrations $(y=0$ and $5 \mathrm{~mm})$ of the limiting reactant $\mathrm{CH}_{4}$ are directly linked to the catalytic reactivity [12], and these quantities are properly captured by the LB model.

As a final accuracy test, the element conservation is also checked. The flux of the element $A$ along a cross section of the channel is

$$
M_{A}=\sum_{j} k_{A j} \int_{0}^{L y} u(y) \rho Y_{j} \frac{m_{A}}{m_{j}} d y,
$$

where $m_{A}$ is the molecular mass of element $A$ and $k_{A j}$ is the number of elements $A$ in species $j$, and $L_{y}$ is the channel height (5 $\mathrm{mm}$ in this application). As an example, the mass flux of the $\mathrm{H}$ element is

$$
M_{\mathrm{H}}=4 \int_{0}^{L y} u(y) \rho Y_{\mathrm{CH}_{4}} \frac{m_{\mathrm{H}}}{m_{\mathrm{CH}_{4}}} d y+2 \int_{0}^{L y} u(y) \rho Y_{\mathrm{H}_{2} \mathrm{O}} \frac{m_{\mathrm{H}}}{m_{\mathrm{H}_{2} \mathrm{O}}} d y .
$$

The element flux must be conserved in each cross section of the channel. Figure 7 provides axial profiles of the relative differences of the element fluxes with respect to the corresponding fluxes at $x=0$. The accuracy of the model is always of the order of $0.1 \%$ at all cross sections and for all elements.

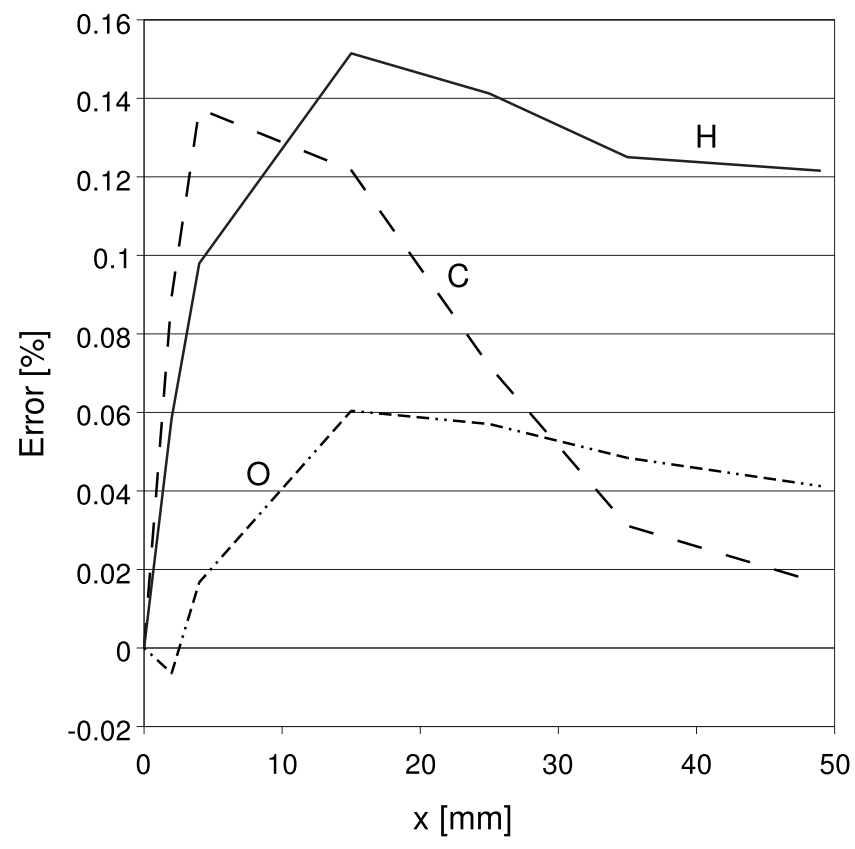

FIG. 7. Relative difference of element fluxes (with respect to the inlet value) as a function of axial distance.

The very good conservation of element fluxes clearly shows the accuracy of the scheme and the employed diffusive boundary conditions.

\section{CONCLUSIONS}

In this paper, we have established a lattice Boltzmann model with catalytic surface reactions. Incorporating reactions at a solid surface was accomplished through the derivation of diffusive wall boundary conditions so as to account for reaction rates. Implementation of realistic chemical reactions is rendered via a coupling of the lattice Boltzmann scheme with the CHEMKIN package libraries. We have also suggested an efficient interpolation scheme for implementation of the lattice Boltzmann algorithm. The developed model is validated with a simulation of a one-step, fourcomponent catalytic methane oxidation reaction in a straight channel. Results are in excellent agreement with a steadystate finite-volume solver. Moreover, the model has been shown to capture finite-rate surface chemistry effects, clearly demonstrating its aptness in practical channel-flow catalytic combustion configurations. Two directions of further studies are currently being explored: Extension of the present approach to more complex boundaries and extension of the current isothermal lattice Boltzmann model to a thermal lattice Boltzmann model recently introduced in Ref. [32].

\section{ACKNOWLEDGMENT}

We acknowledge support from the Competence Center of Energy and Mobility (CCEM) of Switzerland. 
[1] S. Ansumali, I. V. Karlin, S. Arcidiacono, A. Abbas, and N. Prasianakis, Phys. Rev. Lett. 98, 124502 (2007).

[2] P. Asinari, M. C. Quaglia, M. R. von Spakovsky, and B. V. Kasula, J. Power Sources 170, 359 (2007).

[3] Q. Kang, D. Zhang, S. Chen, and X. He, Phys. Rev. E 65, 036318 (2002).

[4] Q. Kang, P. C. Lichtner, and D. Zhang, J. Geophys. Res. 111, B05203 (2006).

[5] S. Succi, A. Gabrielli, G. Smith, and E. Kaxiras, Eur. Phys. J.: Appl. Phys. 16, 71 (2001).

[6] S. Succi, Phys. Rev. Lett. 89, 064502 (2002).

[7] S. Succi, G. Smith, and E. Kaxiras, J. Stat. Phys. 107, 343 (2002).

[8] S. Succi, Lattice Boltzmann Simulation of Thermal Microflows with Heterogenuous Catalysis, in Lecture Notes In Computer Science, Vol. 2657 (Springer, Berlin, 2003), pp. 957-966.

[9] S. Arcidiacono, I. V. Karlin, J. Mantzaras, and C. E. Frouzakis, Phys. Rev. E 76, 046703 (2007).

[10] S. Ansumali and I. V. Karlin, Phys. Rev. E 66, 026311 (2002).

[11] A. Schneider, J. Mantzaras, and S. Eriksson, Combust. Sci. Technol. 180, 89 (2008).

[12] M. Reinke, J. Mantzaras, R. Schaeren, R. Bombach, A. Inauen, and S. Schenker, Combust. Flame 136, 217 (2004).

[13] A. N. Gorban and I. V. Karlin, Physica A 206, 401 (1994).

[14] C. D. Levermore, J. Stat. Phys. 83, 1021 (1996).

[15] S. Arcidiacono, S. Ansumali, I. V. Karlin, J. Mantzaras, and K. B. Boulouchos, Math. Comput. Simul. 72, 79 (2006).

[16] S. Arcidiacono, J. Mantzaras, S. Ansumali, I. V. Karlin, C. Frouzakis, and K. B. Boulouchos, Phys. Rev. E 74, 056707 (2006).

[17] S. Ansumali, S. Arcidiacono, S. Chikatamarla, N. Prasianakis,
A. N. Gorban, and I. V. Karlin, Eur. Phys. J. B 56, 135 (2007).

[18] I. V. Karlin, A. Ferrante, and H. C. Ottinger, Europhys. Lett. 47, 182 (1999).

[19] S. Ansumali, I. V. Karlin, and H. C. Öttinger, Europhys. Lett. 63, 798 (2003).

[20] S. S. Chikatamarla, S. Ansumali, and I. V. Karlin, Europhys. Lett. 74, 215 (2006).

[21] C. Wilke, J. Chem. Phys. 18, 517 (1950).

[22] R. B. Bird, W. E. Stewart, and E. N. Lightfoot, Transport Phenomena (Wiley, New York, 1960).

[23] R. J. Kee, G. Dixon-Lewis, J. Warnatz, M. E. Coltrin, and J. A. Miller, Sandia National Laboratories, Report No. SAND868246, 1996.

[24] M. E. McCracken and J. Abraham, Phys. Rev. E 71, 046704 (2005).

[25] V. Sofonea and R. F. Sekerka, J. Comput. Phys. 184, 422 (2003).

[26] H. Chen, Phys. Rev. E 58, 3955 (1998).

[27] V. Sofonea and R. F. Sekerka, Int. J. Mod. Phys. C 16, 1075 (2005).

[28] V. Sofonea, A. Lamura, G. Gonnella, and A. Cristea, Phys. Rev. E 70, 046702 (2004).

[29] V. Sofonea and R. Sekerka, J. Comput. Phys. 207, 639 (2005).

[30] M. E. Coltrin, R. J. Kee, and F. M. Rupley, Sandia National Laboratories, Report No. SAND90-8003C, 1996.

[31] C. Appel, J. Mantzaras, R. Schaeren, R. Bombach, A. Inauen, B. Kaeppeli, B. Hemmerling, and A. Stampanoni, Combust. Flame 128, 340 (2002).

[32] N. I. Prasianakis and I. V. Karlin, Phys. Rev. E 76, 016702 (2007). 